\title{
RESEARCH
}

Open Access

\section{D flow CMR analysis comparing patients with anatomically shaped aortic sinus prostheses, tube prostheses and healthy subjects introducing the wall shear stress gradient: a case control study}

\author{
Malte Maria Sieren ${ }^{1 *}$ (D), Victoria Schultz ${ }^{1}$, Buntaro Fujita ${ }^{2}$, Franz Wegner ${ }^{1}$, Markus Huellebrand ${ }^{3}$,
} Michael Scharfschwerdt ${ }^{2}$, Hans-Hinrich Sievers ${ }^{2}$, Joerg Barkhausen ${ }^{1}$, Alex Frydrychowicz ${ }^{1}$ and

Thekla Helene Oechtering ${ }^{1}$

\begin{abstract}
Background: Anatomically pre-shaped sinus prostheses (SP) were developed to mimic the aortic sinus with the goal to preserve near physiological hemodynamic conditions after valve-sparing aortic root replacement. Although SP have shown more physiological flow patterns, a comparison to straight tube prosthesis and the analysis of derived quantitative parameters is lacking. Hence, this study sought to analyze differences in aortic wall shear stress (WSS) between anatomically pre-shaped SP, conventional straight tube prostheses (TP), and age-matched healthy subjects) using time-resolved 3-dimensional flow cardiovascular magnetic resonance (4D Flow CMR). Moreover, the WSS gradient was introduced and analyzed regarding its sensitivity to detect changes in hemodynamics and its dependency on the expression of secondary flow patterns.

Methods: Twelve patients with SP (12 male, $62 \pm 9 y$ r), eight patients with TP (6 male, $59 \pm 9 y$ r), and twelve healthy subjects ( 2 male, $55 \pm 6 y r$ ) were examined at 3 T with a 4 D Flow CMR sequence in this case control study. Six analysis planes were placed in the thoracic aorta at reproducible landmarks. The following WSS parameters were

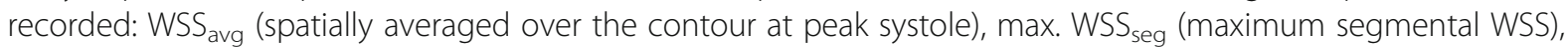
min. WSS seg $_{\text {(minimum segmental WSS) and the WSS Gradient, calculated as max. WSS }}$ seg - min. WSS $S_{\text {seg. }}$ KruskalWallis- and Mann-Whitney-U-Test were used for statistical comparison of groups. Occurrence and expression of secondary flow patterns were evaluated and correlated to WSS values using Spearman's correlation coefficient.

* Correspondence: malte.sieren@uksh.de

'Department for Radiology and Nuclear Medicine, Ratzeburger Allee 160, 23562 Lübeck, Germany

Full list of author information is available at the end of the article

(c) The Author(s). 2020 Open Access This article is licensed under a Creative Commons Attribution 4.0 International License, which permits use, sharing, adaptation, distribution and reproduction in any medium or format, as long as you give appropriate credit to the original author(s) and the source, provide a link to the Creative Commons licence, and indicate if changes were made. The images or other third party material in this article are included in the article's Creative Commons licence, unless indicated otherwise in a credit line to the material. If material is not included in the article's Creative Commons licence and your intended use is not permitted by statutory regulation or exceeds the permitted use, you will need to obtain permission directly from the copyright holder. To view a copy of this licence, visit http://creativecommons.org/licenses/by/4.0/. The Creative Commons Public Domain Dedication waiver (http://creativecommons.org/publicdomain/zero/1.0/) applies to the data made available in this article, unless otherwise stated in a credit line to the data. 
(Continued from previous page)

Results: In the planes bordering the prosthesis all WSS values were significantly lower in the SP compared to the TP, approaching the physiological optimum of the healthy subjects. The WSS gradient showed significantly different values in the four proximally localized contours when comparing both prostheses with healthy subjects. Strong correlations between an elevated WSS gradient and secondary flow patterns were found in the ascending aorta and the aortic arch.

Conclusion: Overall, the SP has a positive impact on WSS, most pronounced at the site and adjacent to the prosthesis. The WSS gradient differed most obviously and the correlation of the WSS gradient with the occurrence of secondary flow patterns provides further evidence for linking disturbed flow, which was markedly increased in patients compared to healthy sub jects, to degenerative remodeling of the vascular wall.

Keywords: 4D flow MRI, Wall shear stress, Valve-sparing aortic root replacement, VSARR, Thoracic aortic aneurysm, Aortic tube prosthesis, Aortic sinus prosthesis, Secondary flow pattern, Hemodynamics, Post-operative outcome

\section{Background}

The established therapy for aneurysms of the aortic bulb and ascending aorta is the implantation of a tubular prosthesis while preserving the aortic valve (valve sparing aortic root replacement, VSARR). Conventional tube prostheses (TP) are known to interfere with the opening of the aortic valve, inducing flow diversions in the aorta and changes in the vessel's anatomy like kinking, post-prosthetic dilatation, and increased transvalvular pressure differences [1-4]. Consequently, anatomically pre-shaped sinus prostheses (SP) were developed to mimic the aortic sinus with the goal to preserve near physiological hemodynamic conditions [2, 5-8]. Yet, the clinical significance of this innovation remains a subject of controversy [9].

Time-resolved 3-dimensional cardiovascular magnetic resonance (CMR) phase contrast imaging (4D Flow CMR) has provided unprecedented insights into hemodynamics of the aorta in vivo, offering visualization of macroscopic blood flow behavior as well as quantification of flow- and velocity-derived parameters [10]. There are several works confirming altered aortic hemodynamics after conventional VSARR [11-14]. Of note, in a previous study using 4D Flow CMR, we could confirm almost physiological flow patterns in the aortic root of the SP compared to disturbed flow in the TP [15]. Nonetheless, distal to both prostheses types, significantly more secondary flow patterns than in healthy subjects were detected $[8,16]$. These secondary flow patterns were only evaluated semiquantitatively on a Likert scale; the study lacked a quantitative parameter to objectively quantify flow abnormalities. While secondary flow patterns are often used as markers for the presence of pathological hemodynamics or even for a specific pathology, e.g. pulmonary artery hypertension $[17,18]$, the effect of these secondary flow patterns on pathological changes of the vessel wall architecture and thus the long-term morbidity of patients exhibiting these flow patterns remain a subject of debate.

We hypothesize that an increase in flow disturbances results in pathological, elevated mechanical stress to the vessel wall. A parameter able to quantify these changes is the wall shear stress (WSS). WSS is a force by blood flow acting tangentially through mechano-transduction on the endothelial cells of the vessel wall. Altered values are linked to pathological vessel wall changes, including remodeling of the vessel wall, atherosclerotic plaque induction, and potential aneurysm growth [19-22]. Recent studies evaluating WSS concentrate on averaged $\left(\mathrm{WSS}_{\mathrm{avg}}\right.$ ) and maximum segmented WSS (WSS $\left.\mathrm{Weg}\right)$ values thereby neglecting minimal values. As both, WSS maxima and minima do affect the vessel architecture $[23,24]$, a comprehensive parameter depicting the gradient between maximum and minimum WSS values potentially provides valuable information to assess hemodynamics and its effects on the vessel wall.

So far, there is only one study assessing WSS in patients who received prostheses with neo-sinus with a relative small number of examined patients [16]. As anatomically pre-shaped SP are specifically designed to re-establish near physiological flow conditions, a comprehensive analysis investigating the interdependencies of pathological flow patterns on WSS and other quantitative flow parameters in SP compared to conventional TP is of fundamental interest, potentially affecting postprocedural patient morbidity and surgical strategies in the long term.

Hence, the aim of this work was to compare quantitative parameters derived from 4D Flow CMR with focus on the newly introduced WSS gradient in patients after implantation of SP to patients who received conventional TP and to age-matched, healthy subjects. In a sub-analysis, the dependency of WSS on the expression of secondary flow patterns was investigated.

\section{Methods}

\section{Study participants}

The study population consisted of three cohorts: Twelve patients with SP, (12 male, 62 $\pm 9 y r$; SP, Uni-Graft W SINUS; Braun, Melsungen, Germany), eight patients with conventional, TP (6 male, $59 \pm 9 y$ r), and twelve age- 
matched, healthy subjects ( 2 male, $55 \pm 6 y r)$. Table 1 provides a comprehensive demographic description of study participants. All subjects were enrolled in this HIPAAcompliant study after approval of the local ethics committee and written informed consent. They were consecutively recruited through the Department for Cardiac Surgery outpatient service and underwent routine follow-up CMR including an additional 4D Flow CMR scan. Patients were recruited dependent on the implanted graft type: patients with TP were scanned and added to a previously presented study collective focused on the semi-quantitative evaluation secondary flow patterns [8]. Previous data were reevaluated for the purpose of the presented hypotheses.

\section{CMR scans}

CMR was performed on a $3 \mathrm{~T}$ scanner (Philips Ingenia Omega dStream, R5.18, Philips Healthcare, Best, The Netherlands) using a 20-channel body surface coil. For 4D Flow CMR acquisitions, a retrospectively electrocardiogram (ECG)-gated, time-resolved, three-dimensional, cartesian phase-contrast CMR sequence with referenced threedirectional velocity-encoding (VENC) was used. Respiratory gating with an acceptance window of $8-12 \mathrm{~mm}$ to achieve a gating efficiency on the order of $60 \%$ was applied. Typical imaging parameters were VENC $=180-200 \mathrm{~cm} / \mathrm{s}$ and time to repeat/time to echo $3.6 / 2.3 \mathrm{~ms}$. Data were acquired with an isotropic resolution of $2.4 \mathrm{~mm}$, reconstructed to $2 \mathrm{~mm}$ and 20 time frames per RR-interval. An effective temporal resolution of 31-67 ms was achieved. Parallel imaging with a sensitivity encoding (SENSE) acceleration factor of 2 resulted in scan times to $\mathrm{PO}$ provided page range in new reference 8 if fine to proceed. Thank you between 9 and 18 min, depending on the individual heart rate and navigator gating efficiency. Eddy currents and Maxwell terms were corrected automatically during offline reconstruction. All imaging parameters were in concordance with the consensus paper by Dyverfeldt et al. [25]. Contrast agent $(1.0 \mathrm{mmol}$ gadobutrol, $0.1 \mathrm{ml} / \mathrm{kg} \mathrm{BW}$, Bayer HealthCare, Berlin, Germany) was administered per clinical routine in all patients and 2 healthy subjects, the flip angle was adapted accordingly to $7^{\circ}$ in studies without and $14^{\circ}$ with contrast agent.

\section{Quantitative data analysis}

Data were transferred to an offline workstation for postprocessing, visualization and quantification. WSS analysis was performed using GTFlow (v3.1.14; GyroTools LLC, Zurich, Switzerland). In six patients, data processing necessitated aliasing correction using PhaseUnwrappingTool (Fraunhofer MEVIS, Bremen, Germany).

Six analysis planes were placed orthogonal to the vessel's axis at reproducible anatomical landmarks: the aortic bulb (bulb), halfway between the valve and the origin of the brachiocephalic trunk in the ascending aorta (AAo), proximal to the origin of the brachiocephalic trunk in the distal ascending aorta (dAAo), between the origins of the left common carotid and the left subclavian artery in the aortic arch (AA), at the location of the ductus diverticulum (DD), and at the level of the first plane in the descending aorta (DAo) (Fig. 1). Vesselcontours were manually segmented using a B-spline algorithm and carefully fitted to the vessel's margins in each time step to account for vessel movement. Correct placement of contours was confirmed via a surface shaded 3D volume angiography based on the velocity-weighted magnitude data (complex difference). Hemodynamic and geometrical parameters stroke volume [ml], maximum flow $[\mathrm{ml} / \mathrm{s}]$, maximum velocity $[\mathrm{cm} / \mathrm{s}]$ and maximum area $\left[\mathrm{mm}^{2}\right]$ were reported. For the WSS analysis, each contour was automatically divided into 8 sub-sections, which were manually aligned to the aortic anatomy (outer curvature [sections 1-2], ventral curvature [sections 3-4], inner

Table 1 Demographics and clinical data of the studies participants

\begin{tabular}{|c|c|c|c|c|c|c|c|c|c|c|}
\hline & \multicolumn{2}{|l|}{ Pat SP } & \multicolumn{2}{|l|}{ Pat TP } & \multicolumn{2}{|l|}{ VOL } & \multirow{2}{*}{$\begin{array}{l}\mathrm{p} \\
\text { all }\end{array}$} & \multicolumn{3}{|l|}{$p$} \\
\hline & Mean \pm SD & range & Mean \pm SD & range & Mean \pm SD & range & & SP vs. TP & Vol vs. SP & Vol vs. TP \\
\hline Age [years] & $55 \pm 15$ & $(26-73)$ & $61 \pm 13$ & $(53-72)$ & $55 \pm 6$ & $(47-69)$ & 0.03 & 0.04 & 0.42 & 0.04 \\
\hline Weight [kg] & $89 \pm 9$ & $(76-100)$ & $83 \pm 15$ & $(78-92)$ & $69 \pm 13$ & $(53-92)$ & 0.53 & 0.81 & $<0.01$ & 0.10 \\
\hline $\begin{array}{l}\text { Gender ratio } \\
\text { [male:female] }\end{array}$ & $11: 1$ & & $8: 1$ & & $2: 10$ & & & & & \\
\hline Height [cm] & $183 \pm 10$ & $(160-200)$ & $178 \pm 6$ & $(174-182)$ & $171 \pm 7$ & $(161-183)$ & 0.10 & 0.33 & $<0.01$ & 0.08 \\
\hline BMI [kg/m2] & $27 \pm 4$ & $(21-32)$ & $25 \pm 6$ & $(23-28)$ & $24 \pm 3$ & $(20-30)$ & 0.44 & 0.98 & 0.30 & 0.38 \\
\hline $\begin{array}{l}\text { Blood pressure } \\
{[\mathrm{mmHg}]}\end{array}$ & $147 \pm 21 / 81 \pm 9$ & $115-180 / 60-95$ & $135 \pm 12 / 85 \pm 7$ & $(125-146 / 80-89)$ & $127 \pm 19 / 80 \pm 10$ & 110-160/70-100) & $0.04 / 0.08$ & $0.24 / 0.27$ & $\mathbf{0 . 0 3 / 0 . 5 0}$ & $0.02 / 0.03$ \\
\hline $\begin{array}{l}\text { Aortic valve, } \\
\text { [tri-/bicuspid] }\end{array}$ & $9 / 3$ & & $8 / 0$ & & $12 / 0$ & & & & & \\
\hline $\begin{array}{l}\text { Heart rate } \\
\text { [bpm] }\end{array}$ & $68 \pm 11$ & $(52-87)$ & $61 \pm 6$ & $(56-63)$ & $63 \pm 9$ & $(49-76)$ & 0.22 & 0.50 & 0.88 & 0.02 \\
\hline $\begin{array}{l}\text { Ejection } \\
\text { fraction [\%] }\end{array}$ & $58 \pm 7$ & $(43-66)$ & $51 \pm 12$ & $(49-58)$ & $65 \pm 3$ & $(61-69)$ & 0.14 & 0.85 & 0.02 & 0.08 \\
\hline
\end{tabular}

Significant differences are marked in bold type. SP Sinus prosthesis, $T P$ tube prosthesis, $B M I$ body mass index, bpm beats per minute 


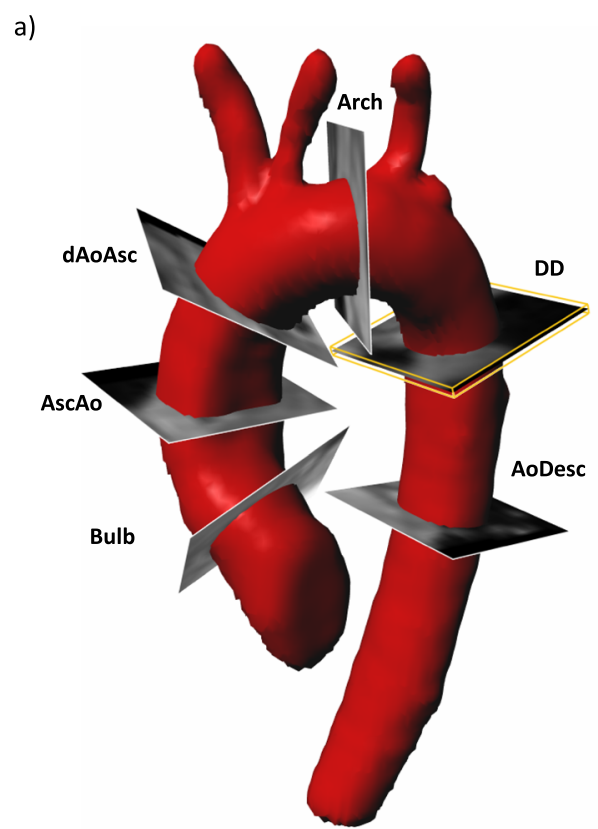

b)
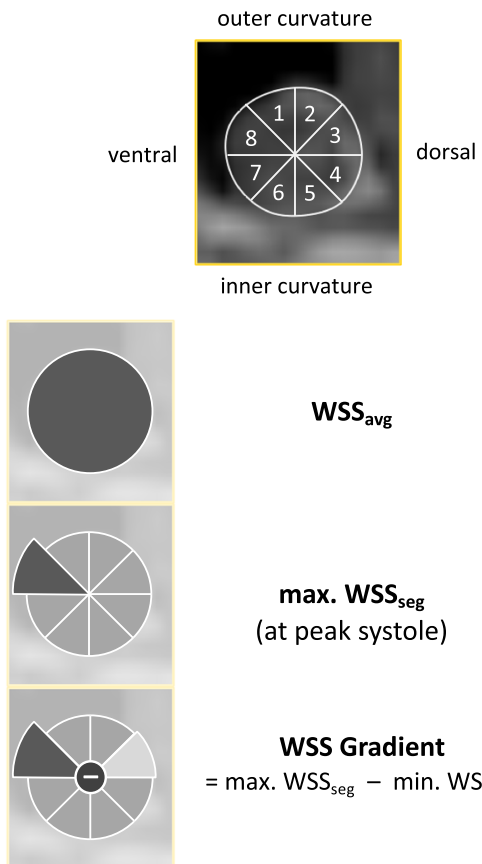

WSS $_{\text {avg }}$

max. WSS $_{\text {seg }}$

(at peak systole)

WSS Gradient

$=\max . \mathrm{WSS}_{\mathrm{seg}}-\min . \mathrm{WSS}_{\mathrm{seg}}$

Fig. 1 Location of contours in the aorta. a Exemplary depiction of contour placement in a 4D Flow dataset of a healthy subject. Contours were placed at reproducible anatomical landmarks at the aortic bulbus (bulbus), halfway between the valve and the origin of the brachiocephalic trunk in the ascending aorta (AoAsc), proximal to the origin of the brachiocephalic trunk in the distal ascending aorta (dAscAo), between the origins of the left common carotid and subclavian artery in the aortic arch (Arch), at the ductus diverticulum (DD) and in the descending aorta (AoDesc). Correct placement was confirmed via a virtual angiography generated from complex difference data. $\mathbf{b}$ Each contour was divided into 8 equally sized sub-segments for wall shear stress (WSS) analysis. To ensure for spatial comparability between planes segments 1 and 2 were orientated to face the outer curvature of the aorta. Recorded WSS parameters were: WSS averaged per plane (WSS avg); maximum WSS per segment (max.

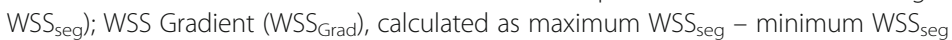

curvature [sections 5-6], dorsal curvature [7, 8]). The following WSS-parameters were recorded in $\mathrm{N} / \mathrm{m}^{2}$ units: Temporal maximum WSS at peak systole, averaged per plane/contour $\left(\mathrm{WSS}_{\mathrm{avg}}\right.$ ); temporal maximum segmental WSS at peak systole (max. WSS $_{\text {seg }}$ ); minimum segmental WSS (min. $\mathrm{WSS}_{\mathrm{seg}}$ ) at the respective timepoint of max. WSS $_{\text {seg; }}$ the WSS gradient $\left(\mathrm{WSS}_{\text {grad }}\right.$ ) was calculated as maximum $\mathrm{WSS}_{\text {seg }}-$ minimum $\mathrm{WSS}_{\text {seg. Location of }}$ contours and systematics of different WSS parameters are illustrated in Fig. 1.

\section{Analysis of blood flow}

Blood flow pattern visualization was achieved via timeresolved pathlines and instantaneous $3 \mathrm{D}$ streamlines emitted up- and downstream from each predefined contour. Data were color-coded with respect to the acquired flow velocity.

Vortices in the sinus and helical flow taking the full diameter of the aortic vessel are examples of physiological, primary flow patterns, which can be found in healthy subjects. Vortices and helices were marked as secondary flow patterns when differing from these physiological primary flow patterns. The presence of secondary vortices was defined as concentric, circular flow in opposition to the main direction of flow within the vessel [26]. A secondary helix was defined as concentric flow in the main flow direction, not filling the complete cross-section of the aorta [27]. Figure 2 depicts exemplary data from a patient study with secondary flow patterns and a healthy subject scan with physiological, undisturbed flow.

Flow patterns were analyzed if they involved one of to the previously defined contours. After detection of a secondary flow pattern in the macroscopic 3D flow field, a scalar map and in plane vectors were created within the adjacent contour to distinguish between helix (helical flow, no backward flow) and vortex (helical flow optional, backward flow). In the next step, distribution of aberrant flow in the vessel's area was evaluated on a scalar map. Secondary flow patterns were graded in semi-quantitative fashion on a scale of $0-3$ as shown before with respect to the diameter of aberrant flow in proportion to the aorta's diameter [15]. The presence and grading of each secondary flow pattern was reported for each contour accordingly. The grading scheme of secondary flow patterns is illustrated in Fig. 3. 
a)

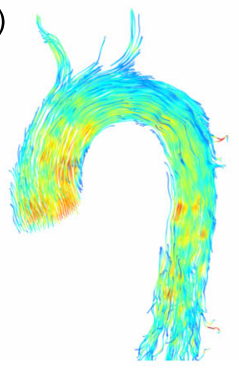

c)

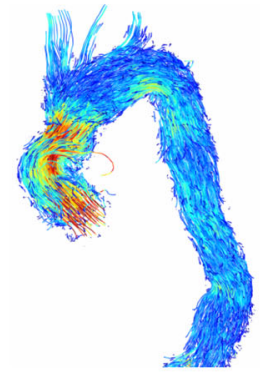

e)

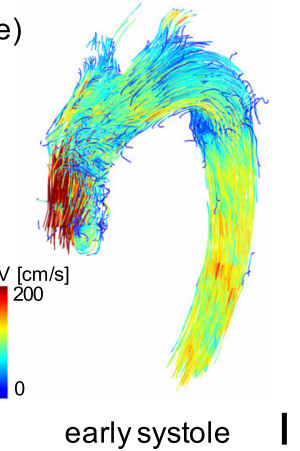

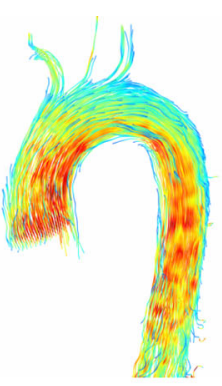
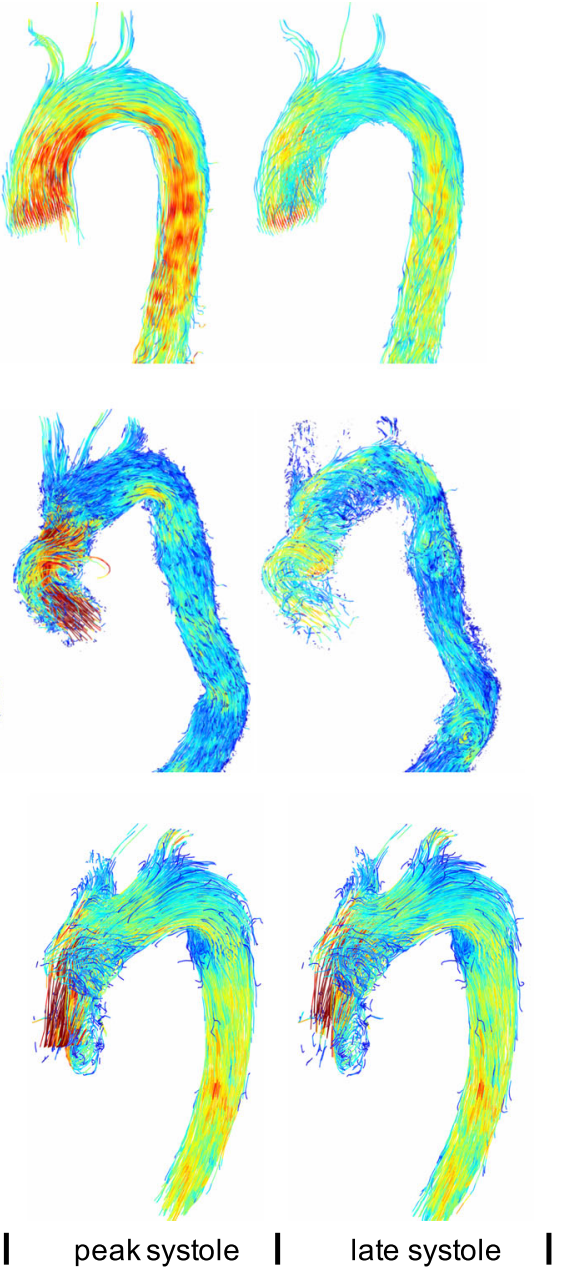

b)
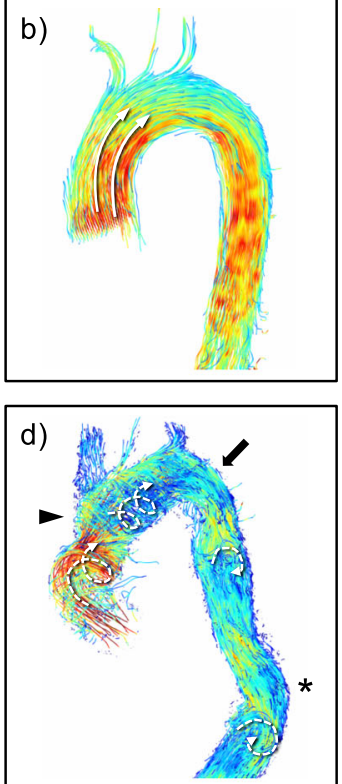

f)

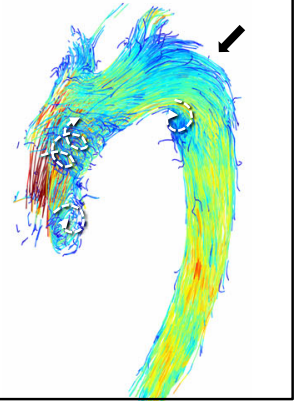

Fig. 2 Comparison between two patients after valve-sparing aortic root replacement and a healthy subject. 4D Flow data of a) a healthy subject, c) a patient with a conventional tube prosthesis (TP) and e) a patient with a sinus prosthesis (SP) replacing the aortic bulb and ascending aorta. Blood flow is visualized starting from the ascending aorta using 3D pathlines, color-coded with respect to flow velocity $(\mathrm{V}$ [cm/s]). Images depict the generation of the flow field from left to right in early systole, late systole and early diastole. Flow patterns in peak systole are traced in $\mathbf{b}$ ), $\mathbf{d}$ ) and $\mathbf{f}$ ). A variety of secondary flow patterns (dotted arrows) form in the aorta of both patients in $\mathbf{d}$ ) and $\mathbf{f}$ ). The subject in $\mathbf{b}$ ) demonstrates undisturbed physiological flow. Both patients show typical changes in the aortic geometry including post-prosthetic dilatation (triangle), postprosthetic kinking (bold arrow) and elongation of the descending aorta (asterisk)

\section{Statistics}

Statistical analyses were performed using SPSS $^{\bullet}$ (Version 25.0. Statistical Package for the Social Sciences, International Business Machines, Inc., Armonk, New York, USA). After testing for normal distribution applying Shapiro-Wilk test values are presented as average \pm standard deviation. To minimize error due to multiple testing differences for WSS, hemodynamic and geometrical parameters between all groups were tested first using Kruskal-Wallis-Test. Subsequently, each patient group (SP and TP) was compared to each other and individually to healthy, aged matched subjects in pairs by means of Man-Whitney-U-Test.
Irrespective of the study participant's status as patient/ volunteer, peak WSS values and the WSS gradient were correlated with the presence and grading of secondary flow patterns per contour by applying Spearman's correlation coefficient. Box plots where calculated to visualize distribution of WSS values depending on the grading of secondary flow patterns.

For the inter-reader comparison, a second observer reevaluated a subset of six individuals, two randomly selected from each group. For WSS values, the intraclass correlation coefficient (ICC; two-way mixed model; absolute agreement; single measurement) with 95\% confidence intervals was computed. For inter-reader 

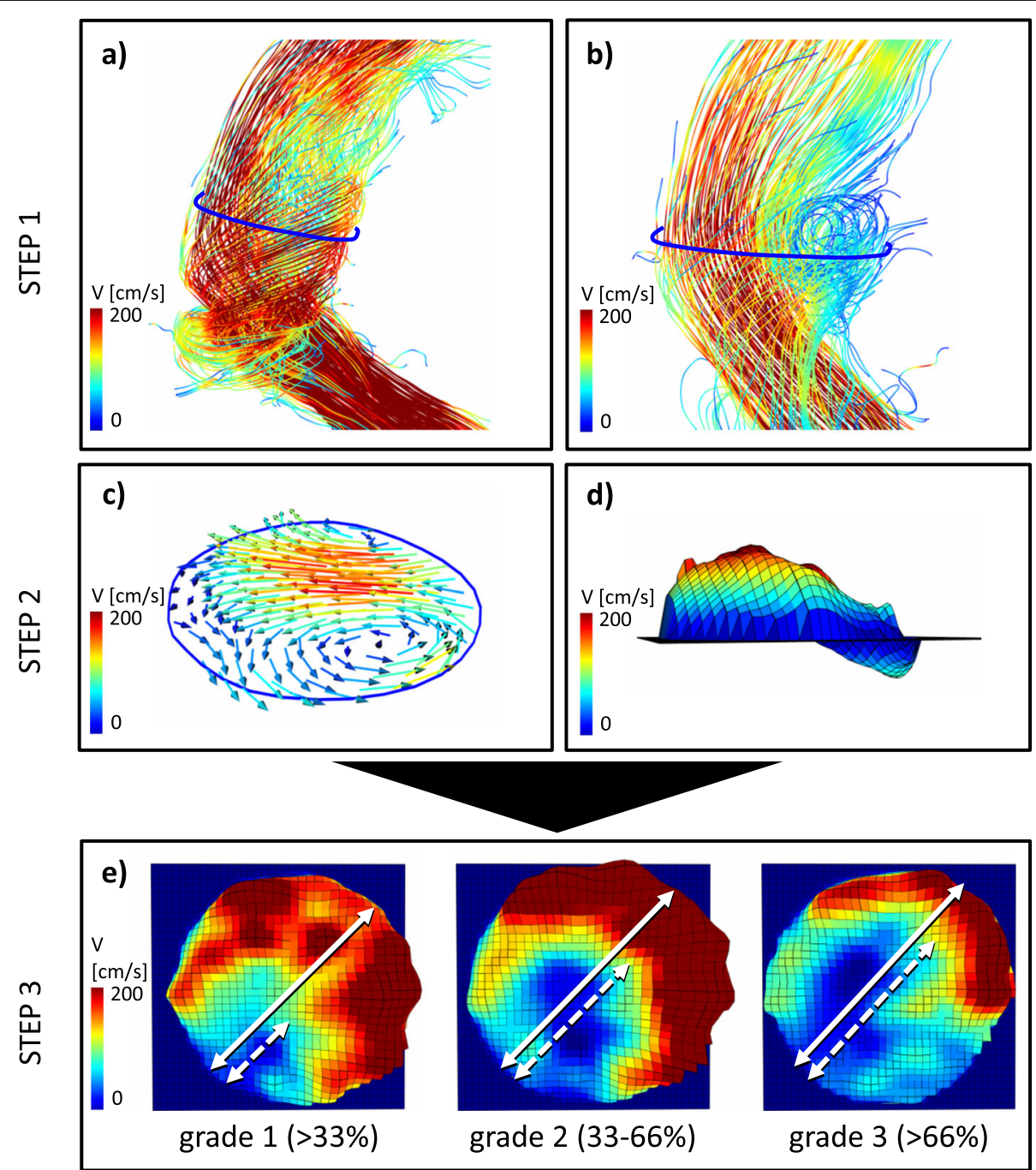

Fig. 3 Grading scheme for secondary flow patterns. In a first step, the flow field was generated using pathlines and 3D streamlines, color-coded with respect to flow velocity $(\mathrm{V}[\mathrm{cm} / \mathrm{s}])$, to detect secondary flow patterns. a demonstrates a secondary helix, $\mathbf{b}$ a secondary vortex in the ascending aorta. Secondly, after a secondary flow pattern was detected, a scalar map and in plane vectors were created to confirm the respective characteristics of $\mathbf{c}$ a helix: helical flow, no backward flow, and $\mathbf{d}$ a vortex: helical flow optional, backward flow. e Lastly, the secondary flow pattern was graded on a scale of 1-3 with respect to the proportion of aberrant flow (dotted arrow) to the aorta's diameter (bold arrow). The grading scheme is based on the method by Oechtering and coworkers [8]

comparison of flow pattern grading Cohen's kappa coefficient was used.

\section{Results}

\section{Quantitative parameters}

Table 2 summarizes the results of the WSS analysis. The WSS gradient was the best parameter to discriminate between groups and showed significantly different values in the ascending aorta and the aortic arch when comparing both prostheses to healthy subjects. In the DAo, only the difference between healthy subjects and patients with TP were statistically significant. Comparing both prostheses against each other, there was a significant difference for WSS gradient in the bulbus and in the ascending aorta. Although values were still elevated in the SP compared to healthy subjects, they were significantly lower than in the TP. Moreover, differences between patients with SP and healthy subjects were less pronounced in the dAAo and DAo than in patients with TP.

WSS $_{\text {avg }}$ and max. WSS the ascending aorta of patients with TP compared to patients with SP and healthy subjects. Both parameters were elevated in patients with TP compared to the other groups. There was a trend for increased values in the aortic bulb in these patients. Min.WSS seg showed no 
Table 2 Analysis of wall shear stress in patients with sinus prosthesis, tube prosthesis and healthy subjects

\begin{tabular}{|c|c|c|c|c|c|c|c|c|c|c|}
\hline \multirow[t]{2}{*}{ WSS $_{\text {avg }}$} & \multicolumn{2}{|l|}{ Pat SP } & \multicolumn{2}{|l|}{ Pat TP } & \multicolumn{2}{|l|}{ Healthy } & \multirow{2}{*}{$\begin{array}{l}p \\
\text { all }\end{array}$} & \multicolumn{3}{|l|}{$p$} \\
\hline & Mean \pm SD & $95 \% \mathrm{Cl}$ & Mean \pm SD & $95 \% \mathrm{Cl}$ & Mean \pm SD & $95 \% \mathrm{Cl}$ & & SP vs. TP & Healthy vs. SP & Healthy vs. TP \\
\hline Bulb & $0.85 \pm 0.35$ & $0.64 ; 1.06$ & $0.96 \pm 0.37$ & $0.66 ; 1.25$ & $0.92 \pm 0.23$ & $0.79 ; 1.05$ & 0.79 & 0.53 & 0.56 & 0.80 \\
\hline Aao & $0.57 \pm 0.13$ & $0.49 ; 0.65$ & $0.82 \pm 0.28$ & $0.59 ; 1.05$ & $0.60 \pm 0.12$ & $0.53 ; 0.66$ & 0.05 & 0.02 & 0.61 & 0.03 \\
\hline dAAo & $0.56 \pm 0.13$ & $0.48 ; 0.63$ & $0.59 \pm 0.16$ & $0.46 ; 0.72$ & $0.65 \pm 0.15$ & $0.57 ; 0.73$ & 0.36 & 0.66 & 0.13 & 0.39 \\
\hline AA & $0.52 \pm 0.13$ & $0.45 ; 0.60$ & $0.50 \pm 0.23$ & $0.32 ; 0.68$ & $0.64 \pm 0.11$ & $0.57 ; 0.70$ & 0.06 & 0.82 & 0.03 & 0.10 \\
\hline DD & $0.51 \pm 0.16$ & $0.42 ; 0.61$ & $0.53 \pm 0.13$ & $0.42 ; 0.64$ & $0.59 \pm 0.15$ & $0.51 ; 0.67$ & 0.45 & 0.82 & 0.25 & 0.38 \\
\hline DAo & $0.68 \pm 0.23$ & $0.55 ; 0.81$ & $0.68 \pm 0.18$ & $0.53 ; 0.83$ & $0.65 \pm 0.12$ & $0.59 ; 0.72$ & 0.97 & 0.99 & 0.72 & 0.70 \\
\hline \multirow[t]{2}{*}{$\underline{\text { max. } W_{S S}}$} & Pat SP & & Pat TP & & Healthy & & $p$ & $p$ & & \\
\hline & Mean \pm SD & $95 \% \mathrm{Cl}$ & Mean \pm SD & $95 \% \mathrm{Cl}$ & Mean \pm SD & $95 \% \mathrm{Cl}$ & all & SP vs. TP & Healthy vs. SP & Healthy vs. TP \\
\hline Bulb & $1.19 \pm 0.40$ & $0.95 ; 1.43$ & $1.73 \pm 0.95$ & $0.97 ; 2.49$ & $1.17 \pm 0.28$ & $0.79 ; 1.05$ & 0.58 & 0.11 & 0.88 & 0.07 \\
\hline Aao & $0.97 \pm 0.25$ & $0.83 \pm 1.12$ & $1.83 \pm 1.02$ & $1.01 ; 2.26$ & $0.82 \pm 0.15$ & $0.74 ; 0.90$ & 0.03 & 0.02 & 0.61 & 0.03 \\
\hline $\mathrm{dAAo}$ & $0.98 \pm 0.28$ & $0.81 ; 1.14$ & $1.16 \pm 0.40$ & $0.84 ; 1.48$ & $0.92 \pm 0.16$ & $0.82 ; 1.01$ & 0.20 & 0.25 & 0.52 & 0.07 \\
\hline AA & $0.80 \pm 0.18$ & $0.69 ; 0.90$ & $0.80 \pm 0.38$ & $0.49 ; 1.10$ & $0.84 \pm 0.15$ & $0.76 ; 0.93$ & 0.08 & 0.99 & 0.53 & 0.71 \\
\hline DD & $0.75 \pm 0.29$ & $0.58 ; 0.93$ & $0.75 \pm 0.19$ & $0.60 ; 0.90$ & $0.80 \pm 0.15$ & $0.72 ; 0.89$ & 0.61 & 0.97 & 0.61 & 0.48 \\
\hline DAo & $0.86 \pm 0.27$ & $0.71 ; 1.02$ & $0.94 \pm 0.19$ & $0.79 ; 1.10$ & $0.83 \pm 0.12$ & $0.76 ; 0.89$ & 0.36 & 0.49 & 0.67 & 0.32 \\
\hline \multirow[t]{2}{*}{ WSS gradient } & Pat SP & & Pat TP & & Healthy & & $p$ & $p$ & & \\
\hline & Mean \pm SD & $95 \% \mathrm{Cl}$ & Mean \pm SD & $95 \% \mathrm{Cl}$ & Mean \pm SD & $95 \% \mathrm{Cl}$ & all & SP vs. TP & Healthy vs. SP & Healthy vs. TP \\
\hline Bulb & $0.75 \pm 0.25$ & $0.61 ; 0.89$ & $1.43 \pm 0.90$ & $0.70 ; 2.15$ & $0.56 \pm 0.12$ & $0.49 ; 0.62$ & $<0.01$ & 0.03 & 0.02 & $<0.01$ \\
\hline Aao & $0.79 \pm 0.23$ & $0.65 ; 0.93$ & $1.64 \pm 1.04$ & $0.80 ; 2.47$ & $0.47 \pm 0.18$ & $0.37 ; 0.57$ & $<0.01$ & 0.02 & $<0.01$ & $<0.01$ \\
\hline dAAo & $0.76 \pm 0.31$ & $0.58 ; 0.94$ & $1.01 \pm 0.30$ & $0.77 ; 1.25$ & $0.47 \pm 0.11$ & $0.40 ; 0.53$ & $<0.01$ & 0.10 & 0.01 & $<0.01$ \\
\hline AA & $0.54 \pm 0.18$ & $0.43 ; 0.64$ & $0.52 \pm 0.35$ & $0.23 ; 0.80$ & $0.37 \pm 0.13$ & $0.30 ; 0.44$ & 0.07 & 0.88 & 0.02 & 0.02 \\
\hline DD & $0.56 \pm 0.27$ & $0.41 ; 0.72$ & $0.57 \pm 0.19$ & $0.42 ; 0.72$ & $0.45 \pm 0.15$ & $0.36 ; 0.53$ & 0.42 & 0.97 & 0.20 & 0.13 \\
\hline DAo & $0.37 \pm 0.19$ & $0.26 ; 0.49$ & $0.5 \pm 0.21$ & $0.34 ; 0.67$ & $0.31 \pm 0.06$ & $0.27 ; 0.35$ & 0.05 & 0.18 & 0.29 & 0.01 \\
\hline \multirow[t]{2}{*}{$\underline{\min . W_{S S}}$} & Pat SP & & Pat TP & & Healthty & & $p$ & $p$ & & \\
\hline & Mean \pm SD & $95 \% \mathrm{Cl}$ & Mean \pm SD & $95 \% \mathrm{Cl}$ & Mean \pm SD & $95 \% \mathrm{Cl}$ & all & SP vs. TP & Healthy vs. SP & Healthy vs. TP \\
\hline Bulb & $0.44 \pm 0.36$ & $0.23 ; 0.56$ & $0.30 \pm 0.16$ & $0.18 ; 0.43$ & $0.61 \pm 0.21$ & $0.49 ; 0.73$ & 0.38 & 0.31 & 0.18 & $<0.01$ \\
\hline Aao & $0.23 \pm 0.10$ & $0.17 ; 0.28$ & $0.19 \pm 0.17$ & $0.05 ; 0.33$ & $0.35 \pm 0.18$ & $0.24 ; 0.45$ & 0.07 & 0.60 & 0.06 & 0.07 \\
\hline dAAo & $0.22 \pm 0.12$ & $0.15 ; 0.29$ & $0.15 \pm 0.11$ & $0.06 ; 0.24$ & $0.45 \pm 0.15$ & $0.36 ; 0.54$ & $<0.01$ & 0.25 & $<0.01$ & $<0.01$ \\
\hline AA & $0.26 \pm 0.15$ & $0.18 ; 0.35$ & $0.28 \pm 0.23$ & $0.09 ; 0.46$ & $0.47 \pm 0.11$ & $0.41 ; 0.53$ & 0.01 & 0.85 & $<0.01$ & 0.02 \\
\hline DD & $0.24 \pm 0.10$ & $0.18 ; 0.30$ & $0.22 \pm 0.09$ & $0.15 ; 0.29$ & $0.36 \pm 0.19$ & $0.25 ; 0.46$ & 0.09 & 0.65 & 0.08 & 0.07 \\
\hline DAo & $0.49 \pm 0.28$ & $0.33 ; 0.65$ & $0.44 \pm 0.20$ & $0.28 ; 0.60$ & $0.52 \pm 0.13$ & $0.44 ; 0.59$ & 0.37 & 0.66 & 0.77 & 0.30 \\
\hline
\end{tabular}

Values for all investigated wall shear stress (WSS) parameters at six measurement contours in the aorta are given. Results of all three groups were tested for significance using Kruskal-Wallis-Test (all). In the column on the far right all three groups were tested in pairs, employing Man-Whitney-U-Test (SP vs. TP, SP vs. Healthy, TP vs. Healthy, respectively). Significant differences are marked in bold type

Aortic bulb [bulb]; ascending aorta (AAo); distal ascending aorta (dAAo); aortic arch (AA); ductus diverticulum (DD); descending aorta (DAo); SP sinus prosthesis; TP tube prosthesis

significant differences between the two prostheses. In comparison to healthy subjects, the values in the aortic bulb, dAAo and AA were significantly lower in patients with both prostheses. With the exception of $\mathrm{WSS}_{\text {avg }}$ in the $\mathrm{AA}$, all other parameters in the course of the vessel did not reach statistical significance.

Table 3 summarizes the results of quantitative flow, velocity and geometrical parameters. Maximum flow was significantly increased in both patient groups on all measurement sites, while maximum velocity only showed significantly higher values in the bulbus and the AAo. In both patient groups, the maximum vascular area was significantly increased in the native vessel distal to the prosthesis. Differences between both patient groups did not reach statistical significance.

\section{Secondary flow patterns and wall shear stress}

Secondary flow patterns were detected in all VSARR patients and five healthy subjects. In spatial relation to the contours, secondary flow formations were found in the AAo in 23 study participants (11 SP; 6 TP; 6 Healthy), in the dAAo in 16 participants ( $8 \mathrm{SP} ; 8 \mathrm{TP}$ ), in the arch in 4 participants ( $1 \mathrm{SP} ; 3 \mathrm{TP})$ and at the DD in 8 participants (2 SP; 3 TP; 3 Healthy). No secondary flow 
Table 3 Analysis of quantitative parameters in patients with sinus prosthesis, tube prosthesis and healthy subjects

\begin{tabular}{|c|c|c|c|c|c|c|c|c|c|c|}
\hline \multirow{2}{*}{$\begin{array}{l}\text { Stroke } \\
\text { volume } \\
\end{array}$} & \multicolumn{2}{|l|}{ Pat SP } & \multicolumn{2}{|l|}{ Pat TP } & \multicolumn{2}{|l|}{ Volunteer } & \multirow{2}{*}{$\begin{array}{l}\mathrm{p} \\
\text { all }\end{array}$} & \multicolumn{3}{|l|}{$p$} \\
\hline & Mean \pm SD & $95 \% \mathrm{Cl}$ & Mean \pm SD & $95 \% \mathrm{Cl}$ & Mean \pm SD & $95 \% \mathrm{Cl}$ & & SP vs. TP & Vol vs. SP & Vol vs. TP \\
\hline Bulb & $99.4 \pm 24.9$ & $84.7 ; 114.1$ & $90.3 \pm 22.9$ & $72 ; 108.6$ & $84.8 \pm 17$ & $75.2 ; 94.5$ & 0.22 & 0.43 & 0.11 & 0.55 \\
\hline AAo & $93.1 \pm 20.6$ & $80.9 ; 105.2$ & $78.9 \pm 13.8$ & $67.9 ; 90$ & $76.4 \pm 13$ & $69 ; 83.8$ & 0.15 & 0.11 & 0.03 & 0.68 \\
\hline dAAo & $87.6 \pm 21.7$ & $74.8 ; 100.4$ & $81.8 \pm 13.7$ & $70.8 ; 92.7$ & $73.5 \pm 16.5$ & $64.1 ; 82.8$ & 0.31 & 0.51 & 0.09 & 0.26 \\
\hline Arch & $62.4 \pm 21.3$ & $49.8 ; 75$ & $65.2 \pm 13.5$ & $54.3 ; 76$ & $53.3 \pm 9.9$ & $47.7 ; 58.9$ & 0.25 & 0.75 & 0.20 & 0.04 \\
\hline $\mathrm{DD}$ & $58.3 \pm 13.8$ & $50.1 ; 66.5$ & $54 \pm 10.8$ & $45.3 ; 62.7$ & $48 \pm 6.9$ & $44.1 ; 51.9$ & 0.17 & 0.48 & 0.03 & 0.14 \\
\hline DAo & $60.1 \pm 13.2$ & $52.3 ; 67.9$ & $52.1 \pm 9.8$ & $44.2 ; 59.9$ & $48.8 \pm 10.3$ & $43 ; 54.7$ & 0.28 & 0.16 & 0.03 & 0.49 \\
\hline \multirow[t]{2}{*}{ Max. flow } & \multicolumn{2}{|l|}{ Pat SP } & \multicolumn{2}{|l|}{ Pat TP } & \multicolumn{2}{|l|}{ Healthy } & $p$ & \multicolumn{3}{|l|}{$p$} \\
\hline & Mean \pm SD & $95 \% \mathrm{Cl}$ & Mean \pm SD & $95 \% \mathrm{Cl}$ & Mean \pm SD & $95 \% \mathrm{Cl}$ & all & SP vs. TP & Healthy vs. SP & Healthy vs. TP \\
\hline Bulb & $486 \pm 114$ & $418 ; 553$ & $459 \pm 53$ & $417 ; 501$ & $379 \pm 99$ & $324 ; 435$ & 0.05 & 0.54 & 0.03 & 0.05 \\
\hline AAo & $490 \pm 92$ & $436 ; 544$ & $447 \pm 39$ & $415 ; 478$ & $344 \pm 75$ & $302 ; 386$ & $<0.01$ & 0.23 & $<0.01$ & $<0.01$ \\
\hline dAAo & $433 \pm 150$ & $344 ; 521$ & $443 \pm 74$ & $384 ; 503$ & $318 \pm 92$ & $266 ; 370$ & 0.04 & 0.85 & 0.04 & $<0.01$ \\
\hline AA & $348 \pm 73$ & $305 ; 391$ & $319 \pm 44$ & $284 ; 355$ & $256 \pm 66$ & $218 ; 293$ & $<0.01$ & 0.34 & $<0.01$ & $<0.01$ \\
\hline $\mathrm{DD}$ & $304 \pm 70$ & $263 ; 346$ & $304 \pm 40$ & $272 ; 337$ & $230 \pm 56$ & $198 ; 262$ & 0.01 & 0.99 & 0.01 & $<0.01$ \\
\hline DAo & $285 \pm 71$ & $243 ; 327$ & $284 \pm 37$ & $254 ; 314$ & $210 \pm 60$ & $176 ; 243$ & 0.05 & 0.97 & $<0.01$ & $<0.01$ \\
\hline \multirow[t]{2}{*}{ Max. velocity } & \multicolumn{2}{|l|}{ Pat SP } & \multicolumn{2}{|l|}{ Pat TP } & \multicolumn{2}{|l|}{ Healthy } & $p$ & \multicolumn{3}{|l|}{$\mathrm{p}$} \\
\hline & Mean \pm SD & $95 \% \mathrm{Cl}$ & Mean \pm SD & $95 \% \mathrm{Cl}$ & Mean \pm SD & $95 \% \mathrm{Cl}$ & all & SP vs. TP & Healthy vs. SP & Healthy vs. TP \\
\hline Bulb & $161 \pm 26$ & $145 ; 176$ & $186 \pm 47$ & $148 ; 224$ & $133 \pm 18$ & $123 ; 144$ & $<0.01$ & 0.15 & 0.01 & $<0.01$ \\
\hline AAo & $148 \pm 42$ & $122 ; 173$ & $148 \pm 45$ & $112 ; 183$ & $97 \pm 17$ & $87 ; 106.4$ & $<0.01$ & 0.99 & $<0.01$ & $<0.01$ \\
\hline dAAo & $128 \pm 35$ & $107 ; 149$ & $154 \pm 44$ & $119 ; 189$ & $120 \pm 79$ & $75 ; 164$ & 0.03 & 0.17 & 0.75 & 0.28 \\
\hline $\mathrm{AA}$ & $104 \pm 21$ & $91 ; 116$ & $108 \pm 34$ & $80 ; 135$ & $93 \pm 21$ & $82 ; 105$ & 0.28 & 0.75 & 0.26 & 0.27 \\
\hline DD & $99 \pm 31$ & $80 ; 117$ & $104 \pm 48$ & $66 ; 143$ & $94 \pm 18$ & $84 ; 104$ & 0.97 & 0.77 & 0.64 & 0.50 \\
\hline DAo & $92 \pm 26$ & $76 ; 107$ & $105 \pm 26$ & $84 ; 126$ & $90 \pm 15$ & $82 ; 99$ & 0.25 & 0.28 & 0.90 & 0.12 \\
\hline \multirow[t]{2}{*}{ Max. area } & \multicolumn{2}{|l|}{ Pat SP } & \multicolumn{2}{|l|}{ Pat TP } & \multicolumn{2}{|l|}{ Healthy } & $p$ & \multicolumn{3}{|l|}{$p$} \\
\hline & Mean \pm SD & $95 \% \mathrm{Cl}$ & Mean \pm SD & $95 \% \mathrm{Cl}$ & Mean $\pm S D$ & $95 \% \mathrm{Cl}$ & all & SP vs. TP & Healthy vs. SP & Healthy vs. TP \\
\hline Bulb & $643 \pm 233$ & $505 ; 781$ & $549 \pm 175$ & $409 ; 689$ & $673 \pm 150$ & $589 ; 758$ & 0.18 & 0.35 & 0.71 & 0.11 \\
\hline AAo & $814 \pm 215$ & $687 ; 941$ & $938 \pm 245$ & $741 ; 1134$ & $736 \pm 182$ & $634 ; 839$ & 0.13 & 0.26 & 0.36 & 0.05 \\
\hline dAAo & $879 \pm 300$ & $702 ; 1057$ & $964 \pm 233$ & $777 ; 1150$ & $567 \pm 145$ & $485 ; 649$ & $<0.01$ & 0.52 & $<0.01$ & $<0.01$ \\
\hline $\mathrm{AA}$ & $738 \pm 268$ & $580 ; 896$ & $729 \pm 206$ & $564 ; 894$ & $462 \pm 126$ & $390 ; 534$ & $<0.01$ & 0.94 & $<0.01$ & $<0.01$ \\
\hline $\mathrm{DD}$ & $624 \pm 291$ & $452 ; 796$ & $675 \pm 151$ & $554 ; 796$ & $408 \pm 102$ & $351 ; 466$ & $<0.01$ & 0.66 & 0.02 & $<0.01$ \\
\hline DAo & $539 \pm 277$ & $375 ; 702$ & $518 \pm 131$ & $413 ; 623$ & $344 \pm 72$ & $303 ; 385$ & $<0.01$ & 0.85 & 0.03 & $<0.01$ \\
\hline
\end{tabular}

Values for all investigated parameters are given at six measurement contours in the aorta are given. Results of all three groups were tested for significance using Kruskal-Wallis-Test (all). In the column on the far right all three groups were tested in pairs (SP vs. TP, SP vs. Healthy, TP vs. Healthy, respectively) by means of Man-Whitney-U-Test. Significant differences are marked in bold type

patterns were detected in the bulb and the DAo in direct spatial relation to the contours. Of the total number of flow patterns analyzed, $42 \%$ were found in patients with SP, $45 \%$ in patients with conventional TP and $13 \%$ in healthy subjects. The flow patterns analyzed in the respective groups were classified into the grading system (Fig. 3; $1=$ low-grade, $2=$ moderate, $3=$ high-grade) as follows: patients with SP $1=14 \% / 2=77 \% / 3=9 \%$; patients with conventional TP $1=29 \% / 2=46 \% / 3=25 \%$; healthy subjects $1=14 \% / 2=86 \% / 3=0 \%$.

Results of Spearman's correlation coefficient can be found in Table 4. Significant correlations were found between the WSS gradient and the grading of secondary flow patterns at all measurement sites as well as for max. $\mathrm{WSS}_{\text {seg }}$ at the level of the AAo. Strong, but insignificant correlations were found for max. $\mathrm{WSS}_{\text {seg }}$ and $\mathrm{WSS}_{\text {avg }}$ in the AAo. The impact of secondary flow patterns on WSS is illustrated in Fig. 4. Box plots demonstrating the distribution of WSS values in relation to the grading of secondary flow patterns in the respective contours are shown in Fig. 5.

Results for the inter-reader comparison of WSS parameters and gradings of flow patterns yielded good to excellent results: ICC for WSS $_{\text {avg }}$ was 0.72 
Table 4 Spearman's correlation coefficients for wall shear stress parameters with secondary flow patterns

\begin{tabular}{llllllll}
\hline & WSS gradient & $p$ & $\underline{\text { max. } \text { WSS }_{\text {seq }}}$ & $p$ & $\underline{\text { WSS }_{\text {avg }}}$ & $p$ \\
\hline Bulb & - & - & - & - & - & - \\
AAo & 0.77 & $<0.01$ & 0.52 & $<\mathbf{0 . 0 1}$ & 0.84 & 0.65 \\
dAAO & 0.77 & $<\mathbf{0 . 0 1}$ & 0.44 & 0.13 & -0.18 & 0.32 \\
AaO & 0.52 & $<0.01$ & 0.20 & 0.27 & -0.20 & 0.27 \\
DD & 0.51 & $<0.01$ & 0.13 & 0.49 & 0.58 & 0.75 \\
DAO & - & - & - & - & - & -
\end{tabular}

Spearman's correlation coefficients for wall shear stress (WSS) parameters at all six measurement contours in the aorta with grading of secondary flow patterns

Significant differences are marked in bold type

[0.52-0.85], for max. WSS seg $^{0.82}[0.69-0.91]$ and for the WSS gradient $0.76[0.57-0.86]$, all $p<0.01$. Cohen's kappa coeffient comparing the grading of secondary flow patterns by both readers was $\mathrm{k}=0.86$.

\section{Discussion}

This study revealed that physiologically shaped SP seem to have a positive impact on aortic WSS compared to conventional TP. We introduced the WSS gradient as a new quantitative parameter to discriminate between different patient groups. Since the WSS gradient is strongly correlated not only with the expression but also with semi-quantitative grading of secondary flow patterns, it provides further evidence to link disturbed flow conditions to degenerative vessel wall changes.

WSS changes in patients were apparent not only at the level of the prosthesis but also in the course of the vessel, potentially inducing long-term pathological altering of the vessel architecture and increasing post-operative morbidity. Significant differences between both prostheses were found in and adjacent to the prostheses. Even if differences did not reach statistical significance, values in the SP were closer to the physiological values of healthy subjects than in the TP underlining the importance of aortic sinuses for hemodynamics. Comparing both prostheses to healthy subjects, significant differences in the AAo were only found for the TP but not the SP. Albeit comparison of different works is hampered by a lack of standardized protocols regarding the evaluation of WSS, the results in the aortic bulb and the AAo are in concordance with a previous study that assessed WSS in a prosthesis with neo-sinuses of a different vendor using a volumetric approach [16]. Arguably, Gaudino and colleagues also reported significant differences for $\mathrm{WSS}_{\mathrm{avg}}$ and maximum WSS in the DAo, which was only partially reproducible in our study.

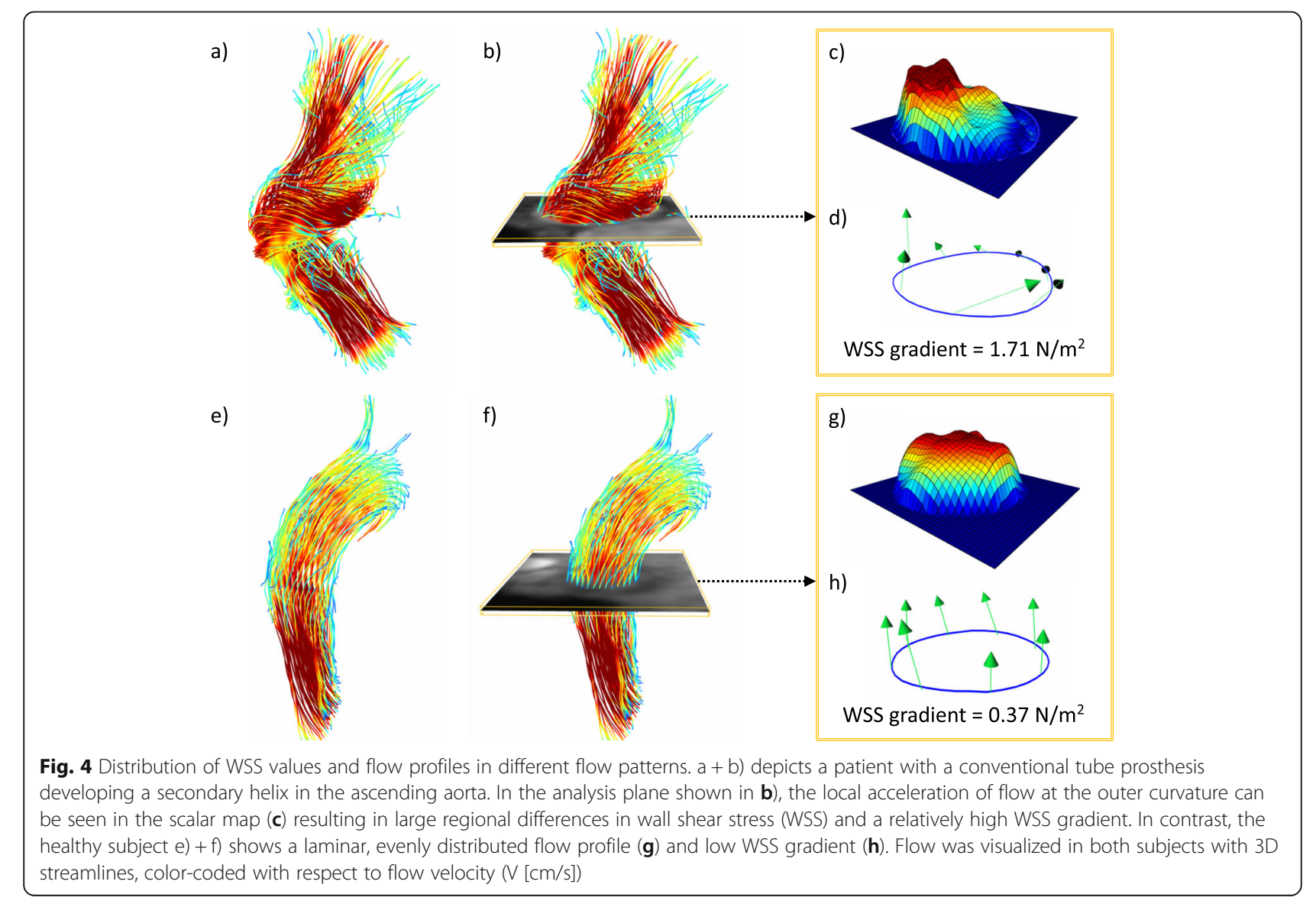




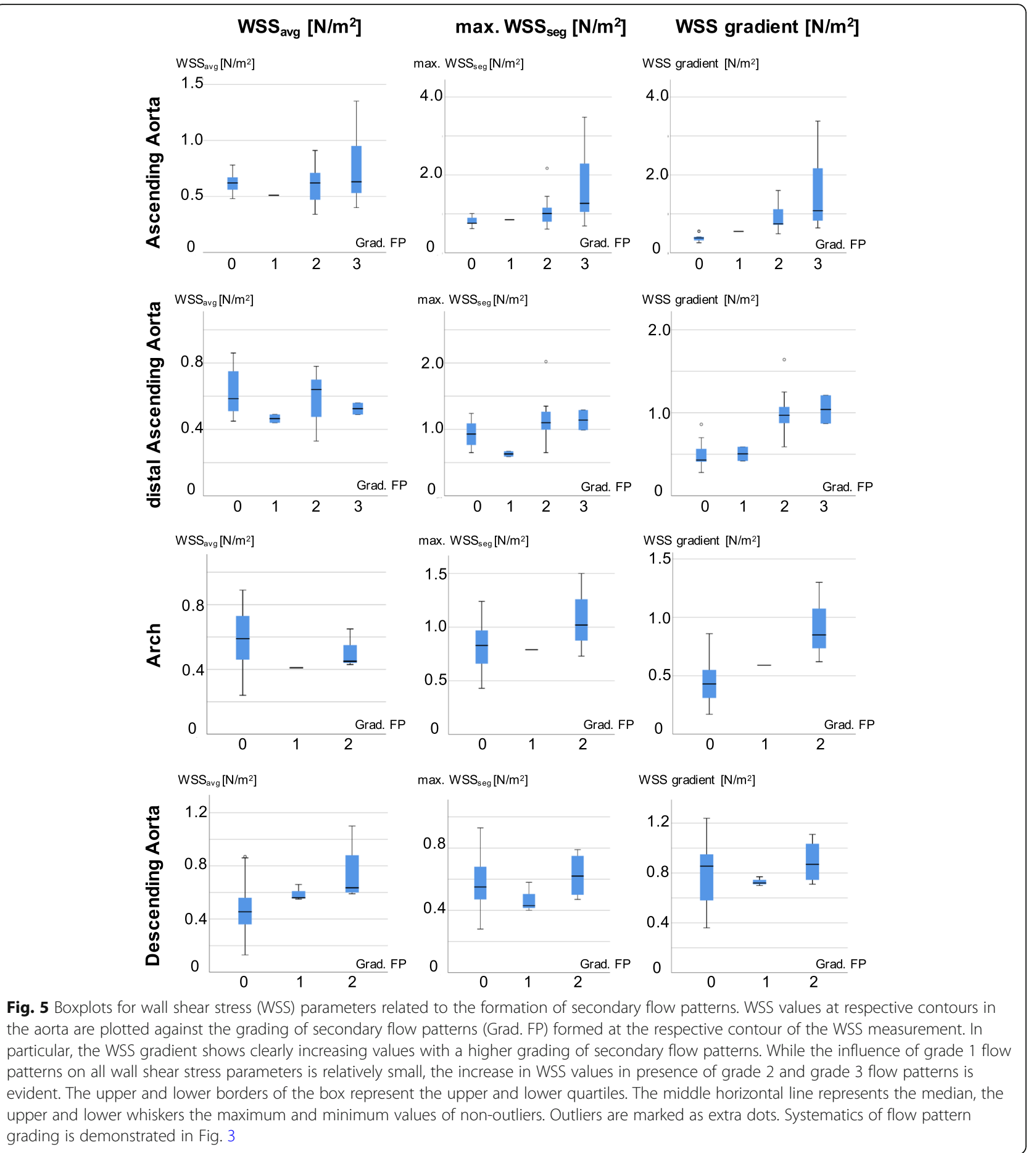

Possible explanations can be found in manufacturerspecific characteristics of the prostheses, various WSS analysis protocols or the small number of patients in each study but remain speculative.

The WSS gradient is a newly introduced parameter, which has not been published before. The concept behind this parameter is to consider not only markedly increased WSS values but also strongly decreased values.
While elevated WSS induces dilatation through increased activity of endothelial nitric oxide synthase and degradation of elastic fibers, reduced WSS is known to induce vessel constriction [23, 24]. Consequently, both, maxima and minima are of pathological relevance promoting endothelial dysfunction and resulting in opposing anatomical changes that have a stronger potential to impair hemodynamics than local WSS alterations 
[24]. However, $\mathrm{WSS}_{\text {avg, }}$ max. $\mathrm{WSS}_{\text {seg, }}$, and min $\mathrm{WSS}_{\text {seg }}$ may only partially portray these changes due to averaging and the exclusion of either peak or minimum values. Both shortcomings are overcome via the WSS gradient. However, one of the disadvantages of 4D flow CMR (e.g. compared to 2D flow CMR), due to the relatively low spatial and temporal resolution, and high signal-to-noise ratio, is an increased inaccuracy in the measurement of maximum and minimum values [25]. We have tried to reduce this drawback by not comparing the maximum and minimum values in individual voxels, but rather in eight regions orientated towards landmarks in the vessel's geometry (Fig. 1), each grouped over several voxels. While the placement of the regions exhibits a potential source of error, good to excellent inter-reader agreement confirms the applicability of this approach.

Although there were marked differences between patients and healthy subjects when analyzing flow visually, established WSS parameters mostly exhibited insignificant tendencies between groups. However, the visually obvious differences in hemodynamics between groups could be quantified with the WSS gradient that was able to depict significant differences between both patient groups and healthy subjects with good inter-reader reproducibility. In both prostheses, the WSS gradient was increased compared to healthy subjects, especially in the dAAo and the AA. Still, measurements in the SP were closer to those of healthy subjects than in patients with TP. While established WSS parameters already indicated differences between the groups, in most cases these did not reach the significance level. This was only achieved by the WSS gradient, which seems to be more sensitive to differences of altered shear forces acting on the vessel wall than established parameters $\mathrm{WSS}_{\text {avg }}$ and max. $\mathrm{WSS}_{\text {seg. }}$ The elevated WSS gradient coincides with an increased number of secondary flow patterns already discussed in a previous paper [8]. Secondary flow patterns are a result of regional accelerations and decelerations of flow and velocity as visualized in the scalar maps in Fig. 2. It seems straightforward that these altered hemodynamic conditions directly influence mechanical forces acting on the vessel wall and it has been hypothesized, that secondary flow patterns are not only a consequence of altered aortic geometry, but also induce its development [28]. The correlations between WSS parameters and a previously introduced semiquantitative grading system of secondary flow patterns [8] provide evidence that an increased number of secondary flow patterns can potentially lead to postoperative aneurysm growth and therefore affect postoperative morbidity. As the WSS gradient was the parameter demonstrating the highest correlation, it may be suitable to quantify and follow-up the effects of disturbed blood flow on the vessel wall. Theoretically, the WSS gradient should be better at identifying vortices characterized by antegrade and retrograde flow in one cross section at the same time in contrast to helical flow. Unfortunately, the study collective was too small to generate the statistical power for such an analysis. Future studies could evaluate the possibility of discrimination between secondary helices and vortices including gradients of other WSS parameters such as axial and circumferential WSS. In both flow patterns there are differently pronounced flow accelerations, which potentially have different effects on the WSS and thus on the vessel wall architecture.

The results for hemodynamic and geometrical quantitative parameters are in concordance with a growing body of literature evaluating patients who underwent VSARR [11-14, 16, 29]. The significantly increased velocities at the level of the prosthesis most likely indicate the reduced compliance of both, the grafts' material and the associated limited Windkessel function of the vessel [30]. This is also an explanatory approach for the significantly increased maximum flow in both prostheses during systole. Nonetheless, maximum velocities were lower in the SP, suggesting a limited reservoir function of the artificial sinus. Moreover, the SP allows for formation of vortices in the sinus [15]. Vortices, in general, have been discussed to preserve kinetic energy $[17,18]$ which may then be released later in the cardiac cycle, partly mimicking a Windkessel effect. Whether the increased maximum area of the aorta in patients is linked to postprosthetic dilatation or the individual's pre-existing vessel dilation remains hypothetical. Lower vessel area distal to the prostheses in SP compared to TP may be interpreted in favor of the SP, however, vessel areas in the descending aorta where slightly larger in patients with SP. Since patients were not examined before surgery, it is possible that any differences existed beforehand and were not related to the type of prosthesis.

Overall, these findings add to the ongoing discussion about the role of the aortic sinuses by offering quantitative proof of improved flow conditions in the anatomically pre-shaped SP. Although no differences in the clinical outcome have been reported so far [31], Guzzardi et al. and Bollache et al. have impressively demonstrated the thinning of elastic fibers at regions of altered WSS in the aorta $[23,24]$. Hence, an effect on the architecture of the aortic vessel wall is highly probable, but may only become evident over the course of several decades. As VSARR is increasingly used in younger patients to maintain function of the native aortic valve $[32,33]$, this may promote the manifestation of late post-operative morbidity.

Despite the positive influence on WSS and hemodynamics as described above, several quantitative parameters still show significant differences between patients with SP and healthy subjects. Besides the limited compliance of graft material, 
the SP is primarily designed to imitate the geometry of the aortic bulb to allow for smooth closure of the aortic valve and perfusion of the coronaries [34, 35]. In the course of the prosthesis and the distal aorta, no measures are taken to improve aortic geometry, resulting in kinking of the prosthesis or at the distal anastomosis as well as post-prosthetic dilatation in both patient groups [8]. To our experience, the postprocedural geometry of the AA substantially affects flow conditions in the DAo [36]. As described in a previous paper, the aortic geometry of our patients, regardless of the prosthesis, differed considerably from healthy subjects [8]. The two patients added to the TP group in this paper also exhibited a more angulated "cubic" aortic anatomy compared to the "round" aortic arch of healthy subjects. This provides a possible explanation for the lack of differences in WSS and other quantitative parameters in the distal aorta. An approach to overcome this limitation is the implantation of so-called $90^{\circ}$ prostheses, which attempt to emulate the natural shape of the AAo. Additional analyses, including these prostheses as well as developing methods to quantify the geometry of the aorta, may further help our understanding of post-procedural hemodynamics after VSARR and improve surgical strategies.

\section{Limitations}

Potential limitations of the study can be seen in the limited size of the patient population. Although of interest, the small number of subjects prevents a correlation analysis of secondary flow patterns and the WSS gradient for each separate group due to the limited statistical power. Factors influencing hemodynamics are manifold and assembling a homogenous patient collective remains a challenge. Since surgery was often performed in an emergency setting (e.g. AAo dissection) with immediate need for action, it was not possible to perform preoperative measurements. As the primary objective of the study was to evaluate the ability of the two different prostheses to achieve close to physiological flow conditions postoperatively, the comparison with an age-matched collective embodying these characteristics seems scientifically sound. As 4D flow CMR was performed after surgery on a voluntary basis, pre-selection bias in patients cannot be excluded. Beside the aorta's characteristics, morphology of the aortic valve may be a factor directly affecting flow conditions [24, 37]; respective analysis would be of great interest but were beyond the scope of this work.

The general limitations of 4D Flow CMR are well known and have been discussed extensively [25]. As investigating hemodynamics post-VSARR with 4D flow CMR is a novel, emerging field, the clinical significance of 4D flow parameters is unclear; the actual effects on the vessel wall remain in part theoretical and larger longitudinal studies are necessary to investigate the clinical correlation of the collected parameters with post-operative morbidity. With $4 \mathrm{D}$ Flow CMR being an evolving method with rapid advances in sequence and post processing techniques, there exists no standardized protocol or cut-off values to evaluate wall shear stress. This hampers comparisons of populations investigated in other studies.

\section{Conclusion}

Physiologically shaped SP seem to have a positive impact on the mechanical forces affecting the aortic wall compared to straight TP in patients after VSARR. Nonetheless, significant differences in WSS, hemodynamic, and geometrical parameters are still present and call for further optimization of surgical techniques and prostheses characteristics. These may include more compliant material and individually tailored grafts, adaptive to the native aortic anatomy.

It should be noted that although established WSS parameters revealed tendentious differences between patients, these did not reach the significant level. We present the novel parameter "WSS gradient", which seems to be more sensitive to changes in WSS than established WSS parameters. Moreover, the WSS gradient correlates with the occurrence and strength of secondary flow patterns. It therefore links secondary flow patterns to pathological, degenerative vessel wall changes potentially affecting postprocedural morbidity. However, while the effect of WSS on the vessel wall is well validated on a histopathological level and different pilot-studies link altered WSS to various pathologies, long-term follow-up studies evaluating the clinical impact of the WSS changes in different patient collectives are warranted.

\section{Abbreviations \\ 4D Flow CMR: Time-resolved 3-dimensional flow cardiovascular magnetic res- onance; AA: Aortic arch; AAo: Ascending aorta; CMR: Cardiovascular magnetic resonance; dAAo: Distal ascending aorta; DAo: Descending aorta; DD: Ductus diverticulum; ECG: Electrocardiogram; SENSE: Sensitivity encoding; SP: Sinus prosthesis; TP: Tube prosthesis; VENC: Velocity-encoding; VSARR: Valve sparing aortic root replacement; WSS: Wall shear stress;

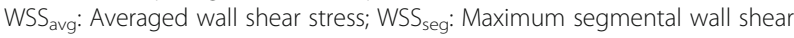 stress}

\section{Acknowledgements}

The authors would like to thank Martina Schroeder for her skillful assistance, Dr. Gerard Crelier and Dr. Hendrik Kooijman-Kurfürst for their continuous support.

\section{Authors' contributions}

Malte Maria Sieren. Analyzed, curated and interpreted the data, performed statistical analysis, wrote, and revised the manuscript. Assisted in designing the study and acquiring data. Victoria Schulz, Franz Wegner. Analyzed the data. Assisted in acquiring the data and revised the manuscript. HansHinrich Sievers, Buntaro Fujita, Michael Scharfschwerdt. Provided background expertise regarding surgical techniques and prostheses. Assisted in designing the study, recruiting patients and revising the manuscript. $\mathrm{HH}$ Sievers provided resources. Markus Huellebrand. Developed the PhaseUnwrappingTool (Fraunhofer MEVIS, Germany) necessary to analyze and to correct aliasing in 4D flow data. Assisted in revising the manuscript. Joerg Barkhausen' Alex Frydrychowicz, Thekla Helene Oechtering. Provided background expertise regarding the CMR sequences and 
acquisitions. All three authors made major contributions designing the study and revising the manuscript. A Frydrychowicz and TH Oechtering supervised the study and assisted acquiring the data. J Barkhausen provided resources. All authors read the manuscript and approved the submission.

\section{Funding}

No funding was acquired to perform this study. Open access funding provided by Projekt DEAL.

\section{Availability of data and materials}

The datasets used and/or analyzed during the current study are available from the corresponding author on reasonable request.

\section{Ethics approval and consent to participate}

All subjects were enrolled in this HIPAA-compliant study after approval of the local ethics committee (Ethics Committee University Luebeck, Ratzeburger Allee 160, GER-23538) and written informed consent. The study ID for ethical approval was 12 - 166A/AZ 12-007.

\section{Consent for publication}

The manuscript does not contain any individual person's data requiring individual consent.

\section{Competing interests}

$\mathrm{HH}$ Sievers receives royalties from B. Braun Melsungen AG. The Department for Radiology and Nuclear Medicine, UKSH Luebeck receives non-financial support from Philips Healthcare, Research Agreement. A Frydrychowicz received Personal Fees from Philips Healthcare, Speaker's Bureau; Bayer Healthcare - Speaker's Bureau. TH Oechtering received Personal Fees from Bayer Healthcare - Speaker's Bureau. All other authors have nothing to disclose with regard to commercial support.

\section{Author details}

${ }^{1}$ Department for Radiology and Nuclear Medicine, Ratzeburger Allee 160, 23562 Lübeck, Germany. ${ }^{2}$ Department for Cardiac and Cardiothoracic Vascular Surgery, University Hospital Schleswig-Holstein, Lübeck, Germany. ${ }^{3}$ Fraunhofer Institute for Digital Medicine MEVIS, Bremen, Germany.

\section{Received: 8 November 2019 Accepted: 8 July 2020}

Published online: 10 August 2020

\section{References}

1. David TE, Feindel CM, David CM, Manlhiot C. A quarter of a century of experience with aortic valve-sparing operations. J Thorac Cardiovasc Surg. 2014;148(3):872-80.

2. Erasmi AW, Sievers HH, Bechtel JF, Hanke T, Stierle U, Misfeld M. Remodeling or reimplantation for valve-sparing aortic root surgery? Ann Thorac Surg. 2007;83(2):S752-6 discussion S85-90.

3. Fries $R$, Graeter $T$, Aicher D, Reul H, Schmitz C, Bohm M, et al. In vitro comparison of aortic valve movement after valve-preserving aortic replacement. J Thorac Cardiovasc Surg. 2006;132(1):32-7.

4. Kallenbach K, Kojic D, Oezsoez M, Bruckner T, Sandrio S, Arif R, et al. Treatment of ascending aortic aneurysms using different surgical techniques: a single-Centre experience with 548 patients. European J Cardio-Thoracic Surg. 2013;44(2):337-45.

5. Salica A, Pisani G, Morbiducci U, Scaffa R, Massai D, Audenino A, et al. The combined role of sinuses of Valsalva and flow pulsatility improves energy loss of the aortic valve. Eur J Cardiothorac Surg. 2016;49(4):1222-7.

6. Maselli D, Weltert L, Scaffa R, Nardella S, Guerrieri Wolf L, De Paulis R. Differences in aortic cusp coaptation between the reimplantation and the remodeling techniques of aortic valve-sparing surgery: an in vitro porcine model study. J Thorac Cardiovasc Surg. 2014;147(2):615-8.

7. Pisani G, Scaffa R, leropoli O, Dell'Amico EM, Maselli D, Morbiducci U, et al. Role of the sinuses of Valsalva on the opening of the aortic valve. J Thorac Cardiovasc Surg. 2013;145(4):999-1003.

8. Oechtering $\mathrm{TH}$, Sieren MM, Hunold P, Hennemuth A, Huellebrand M, Scharfschwerdt $M$, et al. Time-resolved 3-dimensional magnetic resonance phase contrast imaging (4D flow MRI) reveals altered blood flow patterns in the ascending aorta of patients with valve-sparing aortic root replacement. J Thorac Cardiovasc Surg. 2019;418-427 e411.
9. David TE. Aortic valve sparing in different aortic valve and aortic root conditions. J Am Coll Cardiol. 2016;68(6):654-64.

10. Burk J, Blanke P, Stankovic Z, Barker A, Russe M, Geiger J, et al. Evaluation of $3 \mathrm{D}$ blood flow patterns and wall shear stress in the normal and dilated thoracic aorta using flow-sensitive 4D CMR. J Cardiovasc Magn Reson. 2012;14:84.

11. Kvitting JP, Ebbers T, Wigstrom L, Engvall J, Olin CL, Bolger AF. Flow patterns in the aortic root and the aorta studied with time-resolved, 3dimensional, phase-contrast magnetic resonance imaging: implications for aortic valve-sparing surgery. J Thorac Cardiovasc Surg. 2004;127(6):1602-7.

12. Markl M, Draney MT, Miller DC, Levin JM, Williamson EE, Pelc NJ, et al. Timeresolved three-dimensional magnetic resonance velocity mapping of aortic flow in healthy volunteers and patients after valve-sparing aortic root replacement. J Thorac Cardiovasc Surg. 2005;130(2):456-63.

13. Semaan E, Markl M, Malaisrie SC, Barker A, Allen B, McCarthy P, et al. Haemodynamic outcome at four-dimensional flow magnetic resonance imaging following valve-sparing aortic root replacement with tricuspid and bicuspid valve morphology. European J Cardio-Thoracic Surg. 2014;45(5):818-25.

14. Collins JD, Semaan E, Barker A, MCCarthy PM, Carr JC, Markl M, et al. Comparison of hemodynamics after aortic root replacement using valvesparing or bioprosthetic Valved conduit. Ann Thorac Surg. 2015;100(5):1556-62.

15. Oechtering TH, Hons CF, Sieren M, Hunold P, Hennemuth A, Huellebrand M, et al. Time-resolved 3-dimensional magnetic resonance phase contrast imaging (4D flow MRI) analysis of hemodynamics in valve-sparing aortic root repair with an anatomically shaped sinus prosthesis. J Thorac Cardiovasc Surg. 2016;152(2):418-27 e1.

16. Gaudino M, Piatti F, Lau C, Sturla F, Weinsaft JW, Weltert L, et al. Aortic flow after valve sparing root replacement with or without neosinuses reconstruction. J Thorac Cardiovasc Surg. 2019;157(2):455-65.

17. Reiter G, Reiter U, Kovacs G, Kainz B, Schmidt K, Maier R, et al. Magnetic resonance-derived 3 -dimensional blood flow patterns in the main pulmonary artery as a marker of pulmonary hypertension and a measure of elevated mean pulmonary arterial pressure. Circ Cardiovascular Imaging. 2008:1(1):23-30

18. Sieren MM, Berlin C, Oechtering TH, Hunold P, Dromann D, Barkhausen J, et al. Comparison of 4D flow MRI to 2D flow MRI in the pulmonary arteries in healthy volunteers and patients with pulmonary hypertension. PLoS One. 2019;14(10):e0224121.

19. Cheng C, Tempel D, van Haperen R, van der Baan A, Grosveld F, Daemen $\mathrm{MJ}$, et al. Atherosclerotic lesion size and vulnerability are determined by patterns of fluid shear stress. Circulation. 2006;113(23):2744-53.

20. Malek AM, Alper SL, Izumo S. Hemodynamic shear stress and its role in atherosclerosis. JAMA. 1999:282(21):2035-42.

21. Fillinger MF, Marra SP, Raghavan ML, Kennedy FE. Prediction of rupture risk in abdominal aortic aneurysm during observation: wall stress versus diameter. J Vasc Surg. 2003;37(4):724-32.

22. Davies PF, Spaan JA, Krams R. Shear stress biology of the endothelium. Ann Biomed Eng. 2005;33(12):1714-8.

23. Guzzardi DG, Barker AJ, van Ooij P, Malaisrie SC, Puthumana JJ, Belke DD, et al. Valve-related hemodynamics mediate human bicuspid Aortopathy: insights from wall shear stress mapping. J Am Coll Cardiol. 2015;66(8):892-900.

24. Bollache E, Guzzardi DG, Sattari S, Olsen KE, Di Martino ES, Malaisrie SC, et al Aortic valve-mediated wall shear stress is heterogeneous and predicts regional aortic elastic fiber thinning in bicuspid aortic valve-associated aortopathy. J Thorac Cardiovasc Surg. 2018;156(6):2112-20 e2.

25. Dyverfeldt P, Bissell M, Barker AJ, Bolger AF, Carlhall CJ, Ebbers T, et al. 4D flow cardiovascular magnetic resonance consensus statement. J Cardiovasc Magn Reson. 2015;17:72

26. Caro CG, Pedly TJ, Schroter RC, Seed WA. The Mechanics of the Circulation. England: Cambridge University Press; 2012. p. 229-31.

27. Kilner PJ, Yang GZ, Mohiaddin RH, Firmin DN, Longmore DB. Helical and retrograde secondary flow patterns in the aortic arch studied by threedirectional magnetic resonance velocity mapping. Circulation. 1993;88(5 Pt 1):2235-47.

28. Dux-Santoy L, Guala A, Teixido-Tura G, Ruiz-Munoz A, Maldonado G, Villalva $\mathrm{N}$, et al. Increased rotational flow in the proximal aortic arch is associated with its dilation in bicuspid aortic valve disease. Eur Heart J Cardiovasc Imaging. 2019;20(12):1407-17.

29. Galea N, Piatti F, Lau C, Sturla F, Weltert L, Carbone I, et al. 4D flow characterization of aortic blood flow after valve sparing root reimplantation procedure. J Vis Surg. 2018;4:95. 
30. Frydrychowicz A, Berger A, Stalder AF, Markl M. Preliminary results by flowsensitive magnetic resonance imaging after Tiron David I procedure with an anatomically shaped ascending aortic graft. Interact Cardiovasc Thorac Surg. 2009:9(2):155-8

31. De Paulis R, Chirichilli I, Scaffa R, Weltert L, Maselli D, Salica A, et al. Longterm results of the valve reimplantation technique using a graft with sinuses. J Thorac Cardiovasc Surg. 2016;151(1):112-9.

32. Flynn CD, Tian DH, Wilson-Smith A, David T, Matalanis G, Misfeld M, et al. Systematic review and meta-analysis of surgical outcomes in Marfan patients undergoing aortic root surgery by composite-valve graft or valve sparing root replacement. Ann Cardiothorac Surg. 2017:66(6):570-81.

33. Tweddell JS, Earing MG, Bartz PJ, Dunham-Ingles JL, Woods RK, Mitchell ME. Valve-sparing aortic root reconstruction in children, teenagers, and young adults. Ann Thorac Surg. 2012;94(2):587-90 discussion 90-1.

34. Moore BL, Dasi LP. Coronary flow impacts aortic leaflet mechanics and aortic sinus hemodynamics. Ann Biomed Eng. 2015;43(9):2231-41.

35. Katayama S, Umetani N, Sugiura S, Hisada T. The sinus of Valsalva relieves abnormal stress on aortic valve leaflets by facilitating smooth closure. J Thorac Cardiovasc Surg. 2008;136(6):1528-35.

36. Frydrychowicz A, Berger A, Munoz Del Rio A, Russe MF, Bock J, Harloff A, et al. Interdependencies of aortic arch secondary flow patterns, geometry, and age analysed by 4-dimensional phase contrast magnetic resonance imaging at 3 tesla. Eur Radiol. 2012;22(5):1122-30.

37. Rose MJ, Rigsby CK, Berhane H, Bollache E, Jarvis K, Barker AJ, et al. 4-D flow MRI aortic 3-D hemodynamics and wall shear stress remain stable over short-term follow-up in pediatric and young adult patients with bicuspid aortic valve. Pediatr Radiol. 2019;49(1):57-67.

\section{Publisher's Note}

Springer Nature remains neutral with regard to jurisdictional claims in published maps and institutional affiliations.

Ready to submit your research? Choose BMC and benefit from:

- fast, convenient online submission

- thorough peer review by experienced researchers in your field

- rapid publication on acceptance

- support for research data, including large and complex data types

- gold Open Access which fosters wider collaboration and increased citations

- maximum visibility for your research: over $100 \mathrm{M}$ website views per year

At $\mathrm{BMC}$, research is always in progress.

Learn more biomedcentral.com/submissions 